\title{
BLOOD-VESSEL TUMOURS OF THE BRAIN *
}

\author{
By C. Worster-Drought, M.A., M.D., F.R.C.P. \\ Physician, West End Hospital for Nervous Diseases; \\ Physician and Neurologist, Metropolitan Hospital, London, etc.
}

Blood-vessel tumours or angiomata which occur in the brain may be classified under two headings: (I) Malformations of blood-vessels and (2) Haemangioblastomata, or true neoplasms of the elements forming blood-vessels. The former result from a fault in development, and differ from the haemangioblastomata in that they consist of an accumulation of definite, although enlarged, blood-vessels with nervous tissue, intact or compressed, between the vascular loops constituting the angioma.

It is generally agreed that all types of intracranial angiomata are of congenital origin, although often of only slow growth. and development.

Angiomatous malformations include the following conditions:

(a) Telangiectatic angiomata. These are small, mainly capillary, lesions similar to those frequently seen in the skin, and are of no clinical importance as they do not cause symptoms. Such telangiectases are discovered only incidentally during postmortem examinations, and occur most frequently in the floor of the fourth ventricle and in the upper part of the pons.

(b) Capillary angiomata involve the meninges and surface of the brain, and frequently co-exist with similar capillary naevi of the face; and sometimes of the trunk on the same side of the body. This association is known as the Sturge-KalischerWeber syndrome.

Sturge, in 1879 , reported the case of a girl, aged six years, with an extensive capillary naevus, especially of the right side of the face and head, and focal epileptiform attacks starting in the left hand. He concluded that the right side of the brain was involved in the naevoid condition. Kalischer, in 1897 , reported a case of diffuse congenital naevus involving the scalp and face which, on post-mortem examination, showed a lesion described as a blood-vessel tumour composed of capillaries and veins. Parkes Weber, in 1922 , reported the radiographic appearances of the skull of a typical case which showed a large capillary naevus involving especially the left side of the face, and a right-sided upper neurone hemiplegia.

* Based on a postgraduate lecture given at the West End Hospital for Nervous Diseases, London.

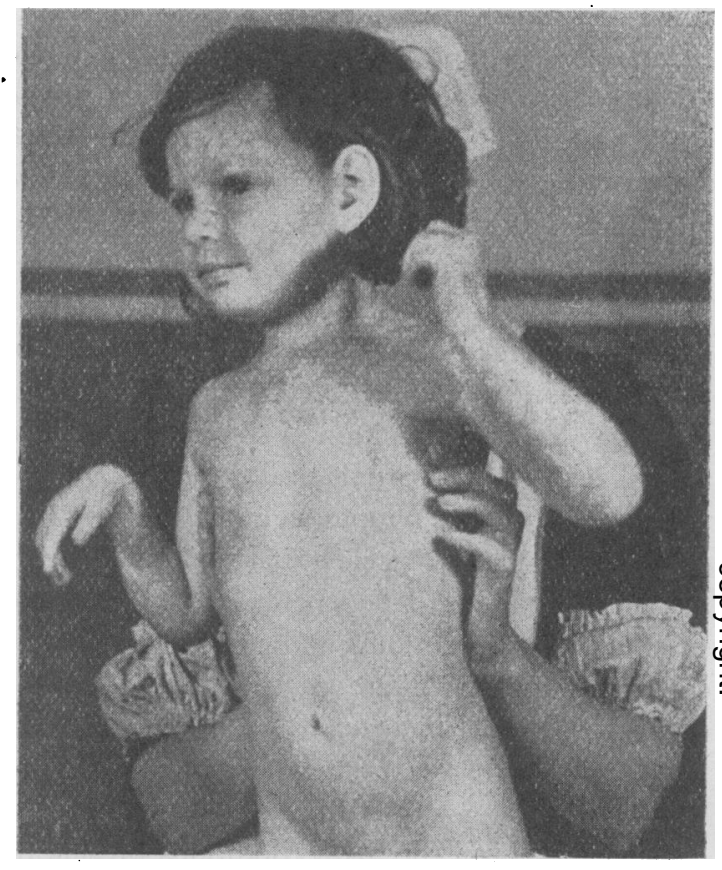

FIG. I.-Sturge-Kalischer-Weber Syndrome. A girl, aged $4 \frac{1}{2}$ years, showing extensive cutaneous naevi on left side of face, upper part of chest and arm. The upper part of the back on the left side, the left leg, both buttocks, and to a lesser extent the right side of the face, also showed scattered naevus formations. The child was mentally deficient and suffered from right-sided hemiplegia and Jacksonian epilepsy. X-ray examination of the skull showed bilateral intracranial calcification, but more pronounced on the left side.

$\mathrm{X}$-ray examination of the skull revealed a more or less calcified lesion on the surface of the left cerebral hemisphere.

The symptoms and signs of Sturge-KalischerWeber syndrome consist in the presence of a facial naevus, with or without similar lesions in other parts of the body (Fig. r), and epilepsy-usually Jacksonian in type and involving the side of the body contralateral to the facial naevus. Not infrequently, a definite hemiparesis is present (Fig. I) with consequent increase in tendon reflexes and an extensor plantar reflex. These conditions may also be associated with buphthalmos (ox-eye or 


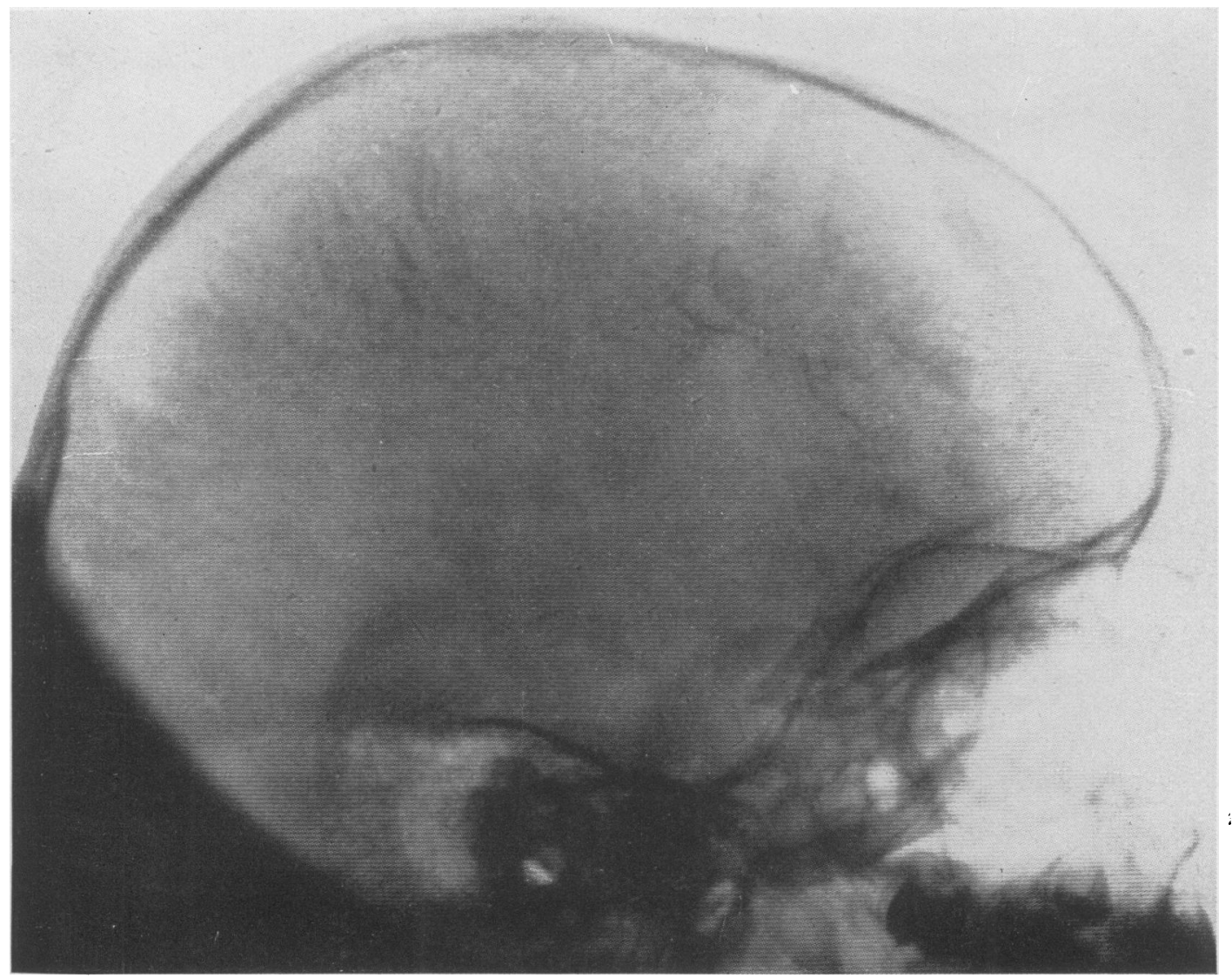

FIG. 2.-Sturge-Kalischer-Weber Syndrome. Skiagram of skull in the case shown in Fig. 1. The calcification occurring in the capillary angioma extends from the frontal region to the superior occipital region and is seen in the form of radial markings, particularly in the parieto-occipital zone. The markings in the posterior frontal region suggest enlargement of the middle meningeal artery.

(Reproduced from the author's commurication by courtesy of the Editor, 'British Medical Fournal ')

congenital glaucoma) on the same side as the main cutaneous naevus, and with mental deficiency. In view of the latter symptom the condition is sometimes termed 'naevoid amentia.' X-ray examination not infrequently reveals the intracranial calcification (Fig. 2) to which reference has already been made.

As judged by the few post-mortem examinations it has been possible to carry out, the intracranial lesion would appear to be mainly a capillary angioma involving the leptomeninges, although some enlarged veins may also be present. The affected cerebral hemisphere is sometimes smaller than that of the opposite side. Very rarely the condition is bilateral; one case was reported by the writer (1948) and N. H. M. Burke, in a subsequent communication, mentioned a case that showed bilateral meningeal angiomata, confirmed by post-mortem examination, but in which the cutaneous naevi were comparatively slight.
An extensive naevus involving one side of the face co-existing with contralateral Jacksonian epilepsy or a hemiparesis, or with both conditions, is sufficient to justify the diagnosis of corticomeningeal capillary (mainly) angioma, even if not revealed by calcification on X-ray examination.

(c) Venous angiomata. Cerebral angiomata consisting entirely of veins are less frequently encountered than other forms of angiomata. Cushing and Bailey ( 1928 ) in a series of $\mathrm{I}, 822$ intracranial tumours verified by operation or autopsy, met with 29 examples of blood-vessel tumour, only six of which were venous angiomata.

With cerebral venous angiomata, symptoms develop at a relatively later age than in either capillary or arterio-venous angiomata.: In most of the recorded cases, the first symptoms appeared in the early twenties. As a rule, the initial symptom is epilepsy-usually focal or Jacksonianindicating that the venous angioma in most cases 


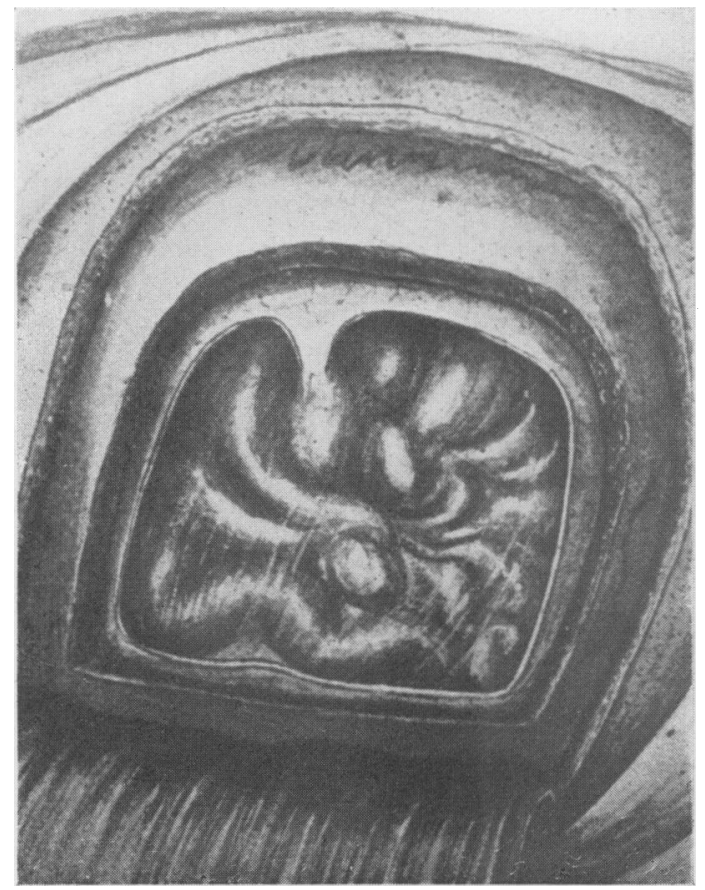

FIG. 3.-Venous Angioma of Cerebrum. Drawing of the operation area exposing a venous angioma involving the right cerebral hemisphere and consisting of enlarged and dilated thin-walled veins. At one point a firm adhesion is seen between an enlarged vein and the dura. The patient, a man aged $3 \mathrm{I}$, had suffered from leftsided Jacksonian epilepsy of four years' duration, with gradually increasing hemiparesis. Following operation (local decompression), the hemiparesis did not progress and the Jacksonian epileptic attacks were reduced in frequency. Death occurred six years later from intracranial haemorrbage, from one of the dilated and thinwalled veins.

(Reproduced from the author's communication by courtesy of the Editor, 'The Lancet')

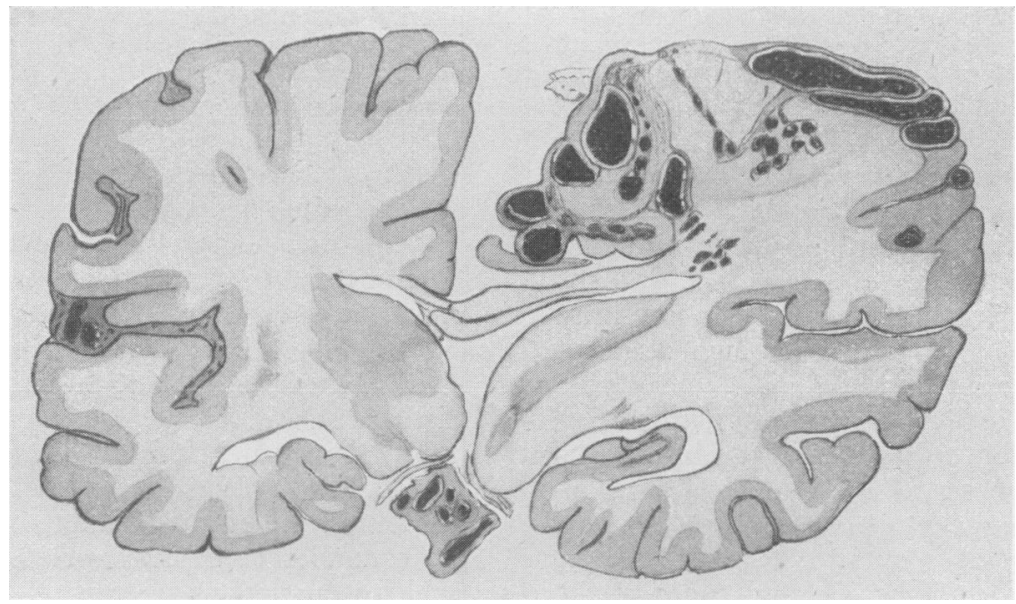

Fsc. 4.-Venous Angioma of Cerebrum. Vertical coronal section through the brain of case illustrated by Fig. 3 showing venous angioma of the right cerebral hemisphere with thin-walled and dilated veins extending from outer to inner aspect of hemisphere. Death occurred from intracranial haemorrhage owing to rupture of one of the enlarged veins.

(Reproduced from the author's communication by courtesy of the Editor 'Yournal of Neurology, Neurosurgery and Psychiatry') 
involves the motor cortex of the cerebrum. Occasional headaches also occur and in other cases there is slow development of hemiparesis. Papilloedema has been recorded as a late manifestation in a few cases, but is more frequent in arterio-venous angioma. Fatal haemorrhage from rupture of one of the thin-walled veins is not infrequent.

Unlike the mainly capillary angioma of the Sturge-Kalischer-Weber syndrome, the purely venous angioma does not tend to calcify and is, therefore, not demonstrable by radiography.

The venous angioma usually consists of large, dilated, thin-walled and non-pulsating veins containing purely venous blood and involving a circumscribed area of the cerebrum, especially the cortex (Fig. 3). According to their anatomical characters, venous angiomata can be classified into: (i) Simple varices - a much enlarged single vein; (ii) Serpentine varices, consisting of several enlarged and tortuous veins; and (iii) Racemose or Cirsoid varices, which are formed of a collection of tangled veins, usually forming a cone-shaped mass, the base of which approximates to the cerebral cortex (Fig. 4), the apex penetrating deeply into the brain and often reaching the lateral ventricle. The terms ' cirsoid,' usually employed by French authors, and ' racemose' by German writers, have often and inappropriately been applied to arteriovenous angiomata, that is, a mainly arterial bloodvessel tumour, whereas a cirsoid or racemose angioma may consist entirely of veins. Cushing and Bailey suggested the term ' angioma venosum,' qualified by racemosum, in contrast to 'angioma arteriale,' similarly qualified.

(d) Arterio-venous angioma, which Cushing and Bailey termed 'angioma arteriale,' consists of a mass of sinuous and tangled pulsating blood-vessels of varying size, both thin-walled and thickened, through which arterial blood flows from enlarged arteries of entry. The angioma is almost invariably aneurysmal in character, showing arterio-venous communications, either from its inception or developing later. The blood escapes from the angioma by one or more greatly dilated veins.

The arterio-venous angioma almost certainly arises from the pial vessels and, although visible in the cortex of the brain beneath the arachnoid, invariably extends deeply into the brain and may even reach the lateral ventricle. Occasionally, the angioma has extensive vascular connections with the dura, while the blood-vessels of the scalp, especially in the temporal region, and the carotid artery, may also be enlarged (Fig. 5a). As with the angioma venosum, the angioma arteriale may be qualified as simple, serpentine or racemose, the latter being the most common.

The angioma arteriale usually becomes sympto- matic in late childhood or in early adult life. Epilepsy, Jacksonian in type, is then an almost constant symptom, and is followed later by hemiplegia and possibly hemianopia. Some cases show exophthalmos, usually on the side of the lesion but occasionally bilateral, with or without swelling of the optic discs, a condition rarely seen with venous angioma. Increased vascularity of the scalp and temporal region, with enlarged and pulsating arteries, hypertrophy and increased pulsation of one or both carotid arteries, with secondary cardiac enlargement, may also occur.

Diagnosis of intracranial angioma. The diagnosis of the Sturge-Kalischer-Weber syndrome has already been discussed. Cerebral venous angiomata may be more common than generally supposed, as if such angiomata involve a so-called silent area of the brain, they may give rise to no symptoms beyond occasional headache and so remain unsuspected. Most recorded cases of cerebral venous angioma have been diagnosed either on exposure of the lesion by surgical operation, carried out on account of focal epilepsy;. or discovered on post-mortem examination. The arterio-venous angioma (angioma arteriale), being usually aneurysmal in character, may give rise to an audible bruit on ascultation of the skull and so reveal its presence; occasionally enlarged bloodvessels may be seen in the scalp, temporal regions, or neck. The co-existence of such enlarged vessels with an audible bruit is pathognomonic of arterio-venous angioma.

In other cases developing Jacksonian epilepsy, with or without mild hemiplegic signs and no papilloedema, and a normal cerebrospinal fluid, an angioma may be suspected. An arterio-venous angioma (angioma arteriale) will often be visible as a partially calcified and diffuse mass on X-ray examination of the skull, but this is not so with a purely venous angioma. In any case, the next step is to carry out angiography by means of the injection of ro cc. of diodone solution, preferably 30 to 40 per cent., into the carotid artery, and by the percutaneous route rather than by open operation. The first exposure is made as the injection proceeds, with two further exposures at intervals of two seconds, in order to obtain both arteriograms and venograms (Fig. 5). Lateral and antero-posterior views shoủld be taken, if possible, to demonstrate the full extent of the angioma.

Treatment of cerebral venous and arterio-venous angioma. Decompression over the lesion may lead to symptomatic improvement and especially to relief of headache, if present, as well as to some reduction in the frequency and intensity of epileptic attacks. In the writer's experience, however, ligature of the carotid artery, on the same side as the cerebral lesion, yields even better 


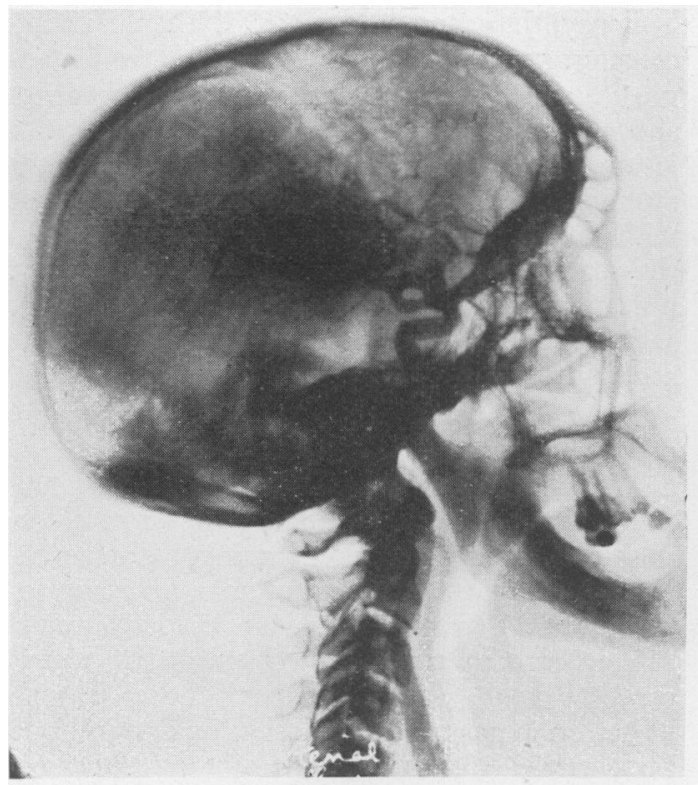

(a)

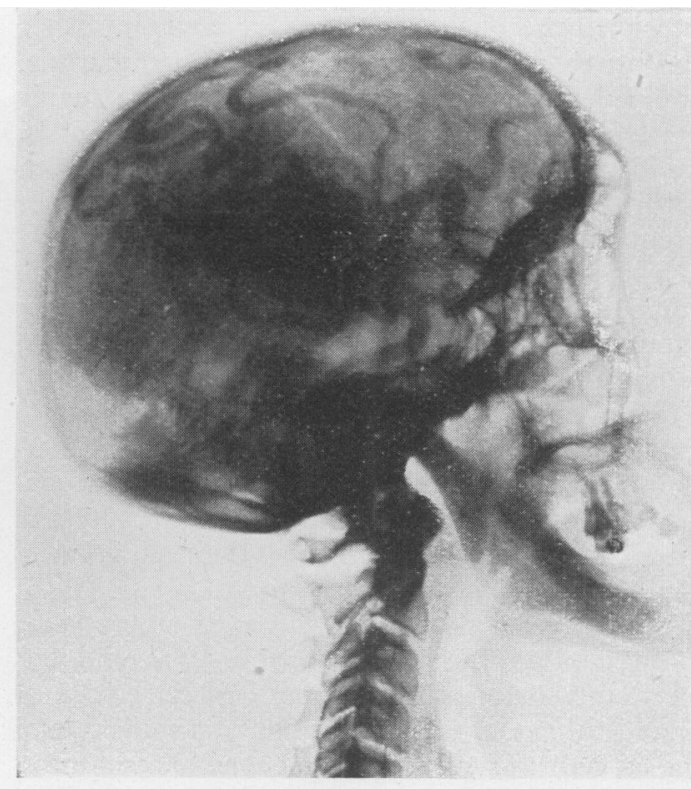

(b)

Fig. 5.-Arterio-Venous Angioma of right Cerebral Hemisphere. (a) Right lateral carotid angiogram taken during the injection of diodone. In addition to the arterio-venous angioma, enlargement of the right internal carotid artery is seen. (b) Angiogram taken by exposure two seconds after $(a)$. The arteriovenous angioma is shown occupying mainly the right Sylvian fissure. The case was that of a man, aged 40, who had been healthy until two years previously. At that time he had an epileptic attack, followed by a second attack ten months ago, since which he had complained of lethargy and lack of concentration. There were no abnormal physical signs, but a bruit was heard over the right eyeball after the angiogram had revealed the lesion. (Included by kind permission of Dr. J. W. D. Bull.)

results and reduces the risk of haemorrhage from the angioma. If exposed by operation, attempts at ligature of the thin-walled veins of a venous angioma may lead to disastrous results and they are best left alone.

Superficial cutaneous angiomata appear susceptible to radiotherapy, and improvement has been reported in some cases of proven cerebral angiomata. Radiotherapy, with or without decompression, may offer some hope of benefit and consequently this form of treatment is worthy of trial.

Haemangioblastoma. The haemangioblastomata are true neoplasms composed of primitive vasoformative cells (angioblasts) which enter into the development of the primordial blood-channels. 'They are congenital in origin, often single but sometimes multiple, and they arise almost exclusively in the cerebellum. Here they are often median tumours, solid or cystic, and appear to arise from the posterior end of the roof of the fourth ventricle.

The haemangioblastoma may be: (a) Capillary, consisting of a small mass of. capillaries or small vascular spaces with a stroma of endothelial cells containing fatty granules, the whole being surrounded by a hyalinized zone; $(b)$ Cellular, containing large epithelial cells with prominent nuclei 윽 and granules, between which run the vascular:channels; (c) Cavernous, composed of large vas- 3 . cular cavities lined with endothelial cells and showing, in the surrounding tissue, epitheloid cells $\frac{}{3}$ with a fine reticulum. These neoplasms often 0 form cysts of varying size containing xanthochromic fluid, in which the angioblastoma is $\frac{D}{0}$ represented by a mural nodule. Occasionally, the cysts in the cerebellum are multiple.

In some cases, a cerebellar haemangioblastoma N is associated with a similar condition in the retina, N the combination often being termed van Hippel's $s_{\sigma}$ disease. In other cases, the angioblastomata are found not only in the cerebellum but also in the $\frac{0}{\Phi}$ spinal cord, kidneys, suprarenals, pancreas and $\stackrel{\oplus}{\rightarrow}$ liver, the syndrome usually being known as 0 Iindau's disease. Several cases showing a heredo- o familial incidence have been reported.

Cerebellar haemangioblastomata should be $\frac{O}{\mathbb{D}}$ treated whenever possible by surgical extirpation. 
When the tumour is cystic, following evacuation of the contents of the cyst, care must be taken to remove the nodule in the cyst wall, as otherwise the symptoms will recur, either from refilling of the cystic cavity or from progressive growth of the angioblastom?.

BIBLIOGRAPHY

BULL, J. W. D. (1049), Proc. Roy. Med., 42, 880

BURKE, N. H. M. (1948), Brit. Med. 7., ii, 53 I.

CUSHING, H., and BAILEY, P. (1928), 'Tumours $\Lambda$ rising from the Blood-vessels of the Brain,' Balliere, London, p. 250.

KALISCHER, S. (1897), Berl. klin. Wochenschr., 30, 1059.

STURGE, W. A. (1879), Trans. Clin. Soc. Lond., 12, 162.
WEBER, 1. PARKlEs (1922), \%. Nemrol. and Psychopathol., 3, 134. WORS'TER-DROUGH'T, $c$., and BALLANCE, C. A. (1922), Lancet, ii, 125 .

WORSTER-DROUGHT, C., and DICKSON, W. E. CARNEGII: (1927), F. Neurol. and Psychopathol., 8, 19.

WORS'TER-DROUGHT, C. (1948), Brit. Med. F., ii, 2 I4.

\title{
OESOPHAGEAL HIATUS HERNIA
}

\section{$\Lambda$ Clinical Study of Forty-Five Cases}

\author{
By Samuel Kaplan, M.D. \\ Department of Medicine, University of Witwatersrand, Yohann'shurg
}

Oesophageal hiatus hernia may be defined as a transdiaphragmatic evisceration of some part of the abdominal contents into the thoracic cavity. 'The herniation occurs through the oesophageal hiatus and the organ that is most commonly involved is the stomach. It is now well established that herniation through the oesophageal hiatus of the diaphragm is not infrequent. 'The complex symptomatology presented by this anatomical variant may, however, render difficulty in its diagnosis.

'The symptoms for which the patients may seek advice may be divided into the following groups :

I. A syndrome of 'dyspepsia.'

2. Pain in the chest stimulating myocardial infarction.

3. Acute gastrointestinal haemorrhage with haematemesis, melaena or both.

4. Symptoms of anaemia probably due to chronic gastrointestinal haemorrhage.

5. A combination of any or all of the above groups.

In addition to the above groups there is a frequent association of other pathological conditions with hiatus hernia. These include cholecystitis with or without cholelithiasis, diverticulosis of the large or small intestine, and chest signs possibly due to aspiration of oesophageal contents.

This report concerns the clinical study of 45 cases of oesophageal hiatus hernia that presented as in and out patients at the Johannesburg General Hospital over a period of 12 months ending March 1949.

Of the 45 cases, 33 were females and 12 males, giving a ratio of females to males of 3 to $r$. The age incidence varied from 35 to 89 years ('Table $\mathrm{I}$ ).
Hiatus herniae of infancy and childhood and traumatic herniae are not included in this study.

In this series the cases are divided into categories according to the following classification introduced by Akerlund (1926):

I. Congenital short oesophagus with partial thoracic stomach.

2. Paraoesophageal hiatus hernia. A portion of the cardiac end of the stomach herniates through the oesophageal hiatus of the diaphragm. 'The oesophagus is of normal length and in its normal position.

3. Oesophagogastric hernia. Both the cardiac end of the stomach and the lower oesophagus herniate through the oesophageal hiatus.

The term 'sliding hernia' is used to designate those cases in which the stomach returns to its normal sub-diaphragmatic position when the patient is erect. Sliding herniae are only demonstrable with the patient supine or in the Trendelenburg position. The term ' congenital short oesophagus' may be a misnomer, although instances of true congenital shortening of the oesophagus are well known (Gross, 1946). In this series, however, instances of short oesophagus were found in elderly patients, the oldest being 89 years of age. Although it is possible that the shortened oesophagus was present from birth, there are other causal factors to be considered. A paraoesophageal or oesophagogastric hernia may, for instance, be associated with post-ulcerative fibrosis, which may shorten the oesophagus in a manner indistinguishable radiologically from a congenital short oesophagus. Allison (1948) has produced evidence to show that short oesophagus is usually an acquired condition, due to defects in the diaphragm which 